\title{
Workplace drug abuse policy: A comment
}

\author{
Richard A. Hirth * \\ Departments of Health Services Management and Policy and Economics, Iniversity of Michigan, \\ 1420 Washington Heights, Ann Arbor, MI 48109-2029, USA
}

Received October 1993; final version received February 1994

Keywords: Drug abuse treatment; Wages

JEL classification: $\mathrm{I} 1 ; \mathrm{J} 3$

McGuire and Ruhm (1993) develop an interesting and policy-relevant model of workplace drug abuse policy. They examine workers' choice to undergo treatment (rehabilitation) for substance abuse and firms' choice of drug-testing policies, concluding that 'labor market incentives will generally lead to too little treatment and too much testing'.

Drug addicts are assumed to have a 'high' probability $(\beta)$ of causing a costly workplace accident while non-addicts are assumed to have a 'low' probability $(\alpha<\beta)$ of causing an accident. Another key assumption is that treatment is perfect. That is, any 'addict' undergoing treatment changes from a 'high' to a 'low' risk with probability one. Although the authors acknowledge that actual treatment regimes are imperfect and discuss how imperfect treatment can be incorporated, they do not appear to recognize the ways in which their qualitative results depend on their simplifying assumption. Allowing partially effective treatment by assuming that the accident risk of treated drug users falls to $\alpha$ with some

\footnotetext{
* Tel. (313)936-1306, fax (313)764-4338, e-mail rhirth@umich.edu.

I would like to thank Michael Chernew, Thomas D'Aunno and an anonymous referee for helpful comments and suggestions. The usual disclaimer applies in full force.
} 
probability p changes some of their conclusions and yields additional insights about the model. ${ }^{1}$

Over two decades of research on the effectiveness of substance abuse treatment programs has uniformly found that relapse is common. One of the most comprehensive and widely cited studies is the Treatment Outcome Prospective Study (see Hubbard et al., 1989). Using an index for multiple drug abuse, less than ten percent of treatment center clients were classified as 'minimal' drug abusers during the year prior to treatment. At a three to five year follow-up, approximately thirty percent were classified as minimal drug users. Using a simplistic definition of treatment success as achieving minimal drug use, this translates to a $p$ on the order of 0.25 . Defined more broadly, long-term indicators of success are not quite as bleak. There was also a shift from more severe patterns of abuse (e.g. cocaine or opioid dominated drug abuse) to less severe patterns (alcohol or marijuana dominated). Thus, some patients who failed to maintain minimal drug use patterns may still have achieved gains. Because multiple and shifting dependencies are ignored, treatment results are more impressive when stated in terms of any particular drug. For example, clients of residential facilities experienced 61 percent declines in the prevalence of 'regular' heroin use. Corresponding declines for regular cocaine, marijuana, and non-medical psychotherapeutic use were 64 percent, 39 percent and 82 percent, respectively. Although the likelihood of regular drug abuse declined, it remained substantial for all classes of drugs. ${ }^{2}$

The qualitative difference between perfect and imperfect rehabilitation in the McGuire and Ruhm model is easy to understand. With perfect rehabilitation, having undergone treatment certifies the worker as being low risk and renders the worker's past drug abuse history irrelevant. When employers can observe treatment, treated addicts are able to send a favorable signal about their accident risk. In equilibrium, McGuire and Ruhm show that fewer addicts pursue rehabilitation than is socially optimal. However, due to the positive signal value of treatment, the extent of undertreatment is lower than it would be if treatment were unobservable. Drug users are more likely to obtain treatment when they can obtain the additional benefit of being able to certify their drug-free status.

By way of contrast, observable but imperfect treatment conveys two useful pieces of information to employers. As in the perfect treatment case considered by

\footnotetext{
${ }^{1}$ This comment focuses on the same 'interesting' equilibrium that McGuire and Ruhm concentrate on. In this equilibrium, no non-addicts undergo rehabilitation and a fraction of addicts chooses treatment.

2 Regular use is defined as daily or weekly use of a substance. In residential programs, regular heroin use declined from $31 \%$ pre-treatment to $12 \%$ at three to five year follow-up. Likewise, regular cocaine use fell from $28 \%$ to $10 \%$, regular non-medical use of psychotherapeutic drugs fell from $50 \%$ to $9 \%$, and regular marijuana use fell from $64 \%$ to $39 \%$. Note that abuse of psychotherapeutic drugs and marijuana were usually not the primary problem leading to admission. Clients of outpatient programs experienced similar declines in usage.
} 
McGuire and Ruhm, treatment signals a reduction in the expected cost of accidents relative to untreated addicts. However, undergoing treatment also signals the worker's history of drug abuse. Because of the possibility of treatment failure (relapse), this information is no longer benign. In particular, the probability of an accident could easily be greater among treated users than in the general untreated population. In this scenario, a wage policy paying each worker his or her expected marginal product penalizes workers for seeking treatment. ${ }^{3}$ The observability of treatment becomes a disincentive to seek help and increases the extent of undertreatment relative to the unobservable treatment case. ${ }^{4}$

The following proposition formalizes these notions. Again, consider McGuire and Ruhm's 'interesting' equilibrium in which non-drug abusers do not seek treatment while some fraction, $\gamma_{\beta}$, of abusers undergo treatment. First, define: $\lambda=$ share of $\beta$-risk workers (drug abusers) initially in the population; $\lambda_{\mathrm{U}}=$ share of $\beta$-risk workers after the fraction $\gamma_{\beta}$ of $\beta$ s get unobserved treatment with a success rate of $p ; \lambda_{\mathrm{T}}=$ share of $\beta$-risk workers among those treated.

Proposition: For any fraction of drug abusers in the general population, $\lambda$, there cxists a probability of treatment success, $p^{*}$, such that trcated drug abusers are better off when employers can observe the treatment decision if the success rate exceeds $p^{*}$. Further when $\lambda$ is small, $p^{*}$ declines in the initial fraction of drug abusers in the population.

Proof: When no non-abusers seek treatment, the fraction of high risk workers in the population after the fraction $p \gamma_{\beta}(p)$ are treated successfully is $\lambda_{U}=\lambda[1-$ $p \gamma_{\beta}(p)$ ]. This is the firm's objective assessment of the probability that a worker presents a high risk of accident when treatment is unobservable. The prevalence of high risks among treated workers is simply the failure rate when only $\beta$ s seek treatment $\left(\lambda_{T}=1-p\right)$. This is the firm's objective assessment of the probability that a treated worker is a high risk when treatment is observable.

In the McGuire and Ruhm model, wage equals expected, net of accident costs marginal product and is strictly decreasing in the firm's belief about the worker's

\footnotetext{
${ }^{3}$ With existing success rates, it is difficult to imagine a job candidate announcing to a prospective employer that he or she has just completed a drug rehabilitation program. The primary exception occurs when the employer already has knowledge of the drug use history, but this is not the case analyzed by McGuire and Ruhm.

${ }^{4}$ In a different context, Burtless (1985) found that targeted wage subsidies actually harmed their intended beneficiaries. A group of AFDC and general assistance recipients were given training in job search skills and then randomly assigned either to search on their own or to receive vouchers that would reimburse employers for half of the worker's first year wages and one quarter of the second year wages. Despite the substantial subsidy, the voucher group was significantly less likely to secure employment. Burtless concludes that by signaling status as a welfare recipient, the vouchers stigmatized workers.
} 
likelihood of causing an accident. Thus, treated abusers are better off under whichever observability regime firms hold the most optimistic beliefs about their accident probability. That is, workers prefer observable treatment when:

$$
\lambda\left[1-p \gamma_{\beta}(p)\right]>1-p
$$

and unobservable treatment when the opposite inequality holds. A success probability $p^{*}$ that equates these expressions leaves workers indifferent between observable and unobservable treatment. $p^{*}$ is implicitly defined by:

$$
\lambda\left[1-p^{*} \gamma_{\beta}\left(p^{*}\right)\right]=1-p^{*}
$$

It is easy to see that (2) can only hold as an equality for $p^{*}$ strictly between 0 and 1 . As shown by McGuire and Ruhm, it is never privately optimal for all drug abusers to obtain therapy. That is, $\gamma_{\beta}\left(p^{*}\right)<1$. Thus, when $p=1$, (1) becomes:

$$
\lambda\left[1-\gamma_{\beta}(1)\right]>0
$$

With perfect rehabilitation, treated workers strictly prefer observability because firms hold more favorable beliefs when they can observe treatment. This is the positive signal value of treatment discussed by McGuire and Ruhm. Conversely, when $p=0$, the inequality in (1) changes direction, becoming $\lambda<1$. For very unsuccessful treatments, workers prefer unobservability. The firm's estimate of the probability that a treated worker presents a high accident risk is greater when treatment is observed (in fact, the firm knows that such a worker is high risk because treatment is useless). Combining these facts, it is clear that (2) can only hold for $p^{*}$ strictly between 0 and $1 .^{5}$

Rearranging (2) defines an identity $F\left(p^{*}, \lambda\right) \equiv 0$ which can be implicitly differentiated for:

$$
\frac{\partial p^{*}}{\partial \lambda}=\frac{p^{*} \gamma_{\beta}\left(p^{*}\right)-1}{1-\lambda\left[\gamma_{\beta}\left(p^{*}\right)+p^{*} \frac{\partial \gamma_{\beta}\left(p^{*}\right)}{\partial p^{*}}\right]}
$$

The numerator is unambiguously negative and the denominator is certain to be positive for small enough fractions of addicts in the general population $(\lambda)$. When the prevalence of drug abuse is low (the case considered by McGuire and Ruhm), $p^{*}$ unambiguously declines in $\lambda$. The lower the prevalence of drug addicts, the

\footnotetext{
${ }^{5}$ For the purposes of this exposition, it is assumed that $p^{*}$ is unique and $\gamma_{\beta}(p)$ is continuous. The arguments made here continue to hold when multiple values of $p^{*}$ satisfy (2) with the following modification. Treated workers are better off under observability for any $p>p^{* \prime}$ where $p^{* \prime}$ is the largest value of $p^{*}$ satisfying (2). Likewise, workers will prefer unobservability at any $p<p^{* \prime \prime}$ where $p^{* \prime}$ is the lowest value of $p^{*}$ satisfying (2). At any $\mathrm{p}$ such that $p^{* \prime \prime}<p<p^{* \prime}$, treated workers are better off under whichever regime firms hold the most optimistic beliefs about their accident probability. A similar extension of the argument covers the case in which $\gamma_{\beta}(p)$ is discontinuous.
} 
more successful rehabilitation must be for treated workers not to prefer hiding their treatment status in order to pool with non-addicts.

McGuire and Ruhm treat the observabililty of treatment as exogenous. However, the partially effective treatment scenario can endogenize the observability regime. It seems plausible to hypothesize that workers can credibly demonstrate that they have completed treatment. For example, they might show the employer letters from physicians or representatives of accredited treatment facilities, bills, canceled checks or insurance explanation-of-benefits forms. Given the confidentiality of the provider/patient relationship, it is also plausible to hypothesize that workers can hide their treatment history from employers if they choose to keep this information private. ${ }^{6}$

On the basis of the probability of relapse and the prevalence of treated and untreated addicts in the population, treated workers can decide whether or not revealing their treatment status is advantageous. When treatment is highly successful, the observable treatment regime is the equilibrium outcome as the positive signal value of therapy outweighs the negative effect of revealing drug abuse history. Alternatively, if the failure rate is substantial, the cost of revealing past drug abuse exceeds the worker's benefit from the positive treatment signal and the unobservable treatment regime pertains. ${ }^{7}$ If the prevalence of drug abusers in the population is very low, rehabilitation must be nearly perfect before treatment provides a favorable signal. In a population initially containing many drug abusers, even poor treatment offers workers some opportunity to distinguish themselves from the chemically dependent masses. Under realistic drug abuse prevalence and treatment success rates, it seems very unlikely that treated workers would bencfit from revealing their status.

The observation that gaining employees' trust has been a major obstacle in establishing employee assistance plans (EAPs) for drug abusers can only be explained in an imperfect treatment environment. Workers often hesitate to take part in firm-based substance abuse programs because they are revealing information that the employer may use against them. Even if workers somehow develop enough trust to come forward, McGuire and Ruhm's observable treatment model may not be relevant. With high relapse rates, firms can only establish trust by refraining from making downward wage revisions when workers voluntarily reveal their treatment status. A more complicated model with repeated interactions

\footnotetext{
${ }^{6}$ Because of these same confidentiality requirements, untreated workers cannot credibly communicate that they have not undergone treatment.

${ }^{7}$ Some workers may have above or below average probabilities of success. Workers who believe their success probability exceeds $p^{*}$ may reveal their treatment status in an attempt to communicate their optimistic prognosis. However, this scenario seems unlikely. Workers with lower likelihoods of success may wish to pool with the self-revealed treated addicts. More importantly, empirical evidence indicates that it is very difficult to predict which individuals will relapse. For example, McLellan et al. (1993) find that only a small proportion of the variance of the six month success in reducing drug abuse can be explained by an impressive set of independent variables.
} 
between workers and firms would be required to give firms an incentive to nurture a reputation for trustworthiness. If trust can be established, EAPs may decrease the extent of undertreatment by lowering the effective cost to the employee as workers face lower travel and search costs as well as lower out-of-pocket costs if firms subsidize the program.

Explicitly considering the imperfect treatment case not only adds realism to the model, but it also clarifies an interesting 'second best' result. In McGuire and Ruhm's original formulation, treatment confidentiality (unobservability) reduces welfare. A policy requiring disclosure of treatment records to firms would make workers better off. Further, workers would be more likely to seek treatment under mandated disclosure.

Imperfect treatment can yield the opposite conclusion. Confidentiality prevents wage policies from discouraging workers from obtaining efficient treatment by allowing treated high risk workers to pool with low risk workers whenever it is beneficial not to reveal drug abuse status. If treatment is observable (e.g. if disclosure is mandated), drug abusers can mimic low risk workers only if they remain untreated. Due to moral hazard, the extent of undertreatment relative to the social optimum worsens. This conclusion is diametrically opposed to McGuire and Ruhm's finding for the perfect treatment case. Private information can actually be welfare-enhancing and a policy requiring disclosure would be detrimental. Provided workers can credibly communicate that they have received therapy, it becomes clear that treatment confidentiality is an optimum policy. If treatment is so successful that treatment provides a favorable signal about accident risk, treated workers will voluntarily disclose their status to firms. Presumably, insurance records and authorizations for release of medical records would ensure the credibility of these claims. Alternatively, if treated drug abusers have substantial relapse rates, the ability to hide the fact of treatment from employers prevents wage and hiring policies from discouraging drug abusers from seeking help. Under current success rates, it is difficult to imagine that many workers would voluntarily disclose that they have undergone substance abuse therapy. The only notable exceptions would seem to those whose drug abuse history has already been discovered. Because their employers already hold negative beliefs about their accident rates, even imperfect treatment improves the firm's prior beliefs.

\section{References}

Burtless, G., 1985, Are targeted wage subsidies harmful? Evidence from a wage voucher experiment, Industrial and Labor Relations Revicw 39, 105-114.

Hubbard, R.L. et al., 1989, Drug abuse treatment: A national study of effectiveness (University of North Carolina Press, Chapel Hill, NC).

McGuire, T.G. and C.J. Ruhun, 1993, Workplace drug abuse policy, Journal of Health Economics 12 , $19-38$.

McLellan, A.T. et al, 1993, Similarity of outcome predictors across opiate, cocainc and alcohol treatmentc role of treatment services. Iournal of Consulting and Clinical Psycholngy, forthonming. 